\title{
Instant Human Face Attributes Recognition System
}

\author{
N.Bellustin \\ ${ }^{1}$ Research Institute of Radio \\ Physics, Nizhny Novgorod, Russia
}

Kovalchuck, A. Telnykh, O. Shemagina and
V.Yakhno
${ }^{3}$ Institute of Applied Physics, Nizhny Novgorod, Russia,

\begin{abstract}
The objective of this work is to provide a simple and yet efficient tool for human attributes like gender, age and ethnicity by the human facial image in the real time image as we all aware this term that "Real-Time frame rate is a vital factor for practical deployment of computer vision system". In this particular paper we are trying to presents the progress towards face detection and human attributes classification system. We have developed an algorithm for the classification of gender, age and race from human frontal facial image As the basis of the classifier proposed algorithm uses training set neuron receptors that process visual information a study of the several variants of these classifiers and shows the principal possibility of sex determination, assessment of a person's age on a scale (adult children) and recognition of race by using the neuron-like receptors.
\end{abstract}

Keywords- Gender recognition; Age recognition; Ethnicity recognition; MCT; AdaBoost; attributes classifier.

\section{INTRODUCTION}

The human brain is a dynamical system whose state evolves with time. Stimuli from the outside world are by cortex neuron processed and transformed, with inner human brain models compared and then fed-back throughout the brain. The complex dynamical interaction with these inner models, which human foregoing experience accumulate, provide right making decision and true classify objects of different types. The human brain was million years constructed and tuned, and now it is arguably the best known classifier - it can learn complex

Classification tasks fast and surely. This high efficiency of natural biological system of image processing have long motivated researchers to seek to best understanding of the brain operation and to construct artificial electronic systems with these high characteristics.

One the main step in this direction is to construct an "Attribute Classifier" which classifies the human attributes like gender, age, race and emotional state from the facial image with the efficacy comparable - the present paper is devoted to this interesting and complicated problem. There is well known that human faces convey lots of information and the high-level semantic information about the identification of such attributes. Automatic human attributes classification based on human face image aims at recognizing the attributes according to face appearance in images. In this paper we present a system for

\author{
Y. Kalafati ${ }^{2}$ \\ ${ }^{2}$ Institute of Radio Engineering and Electronics RAS, \\ Moscow, Russia,
}

\author{
Abhishek Vaish, Pinki Sharma, Shirshu Verma ${ }^{4}$ \\ ${ }^{4}$ Indian Institute of Information Technology, Allahabad, \\ INDIA
}

face detection and recognition of the attributes of a person, which is based on a unified approach to the recognition of some types of attributes that can be associated with the face of the person - gender, age, race, presence of beard and mustache, glasses and so on. From the experiments that our approach can be used for attributes recognition by the human face at realtime.

\section{RELATED WORK}

In order to interact socially, we must be able to process human faces in diversity of ways. There is vast amount of literature on cognitive psychology attesting capabilities of human at identifying faces .Most of work to date has been on identity verification like gender, age and emotional state of human face, and only few work have been concerned with combination of human attributes (age, gender and ethnicity) classification in real-time .

\section{A. Age Classification-}

Age classification of person from the digital image is still challenging task of the image analysis. Numerous authors have highlighted this research question. However, authors have not achieved much accuracy like human beings.

Yi-Wen Chen, Meng-Ju Han,Kai-Tai Song and Yu-Lun $\mathrm{Ho}[1]$ presented experimental analysis of Classification of human- age using facial features. 52 feature point extracted by using Lucas- Kanade image alignment method. These feature points and corresponding located facial area are used to build an active appearance model (AAM). (AMM based feature points).In this paper they used different image processing skill to express features like gray level image ,edge image, gray image with edge image and horizontal image. These features are sent into a support vector machine (SVM) to estimate the level of age group and achieved $87 \%$ accuracy. Experiment on FERET and FG-NET Database.

Asuman GÜNAY [2],presented Estimation of the age exactly for the security system.LBP (Local Binary pattern and histogram of these pattern, LBP histogram are extracted and concatenated into a feature vector. In the classification phased, minimum distance, nearest neighbor and k-nearest neighbor classifier were used for classification and achieved $80 \%$ system performance for age estimation. Experiment on FERET and FG-NET Database. Jian-Gang Wang[3], introduced novel Age 
categorization method to classify face images into several Agegroup. They considered age estimation as a multiclass problem. Aging feature extracted using Gabor feature, LBP, Feature fusion and Adaboost is used for the boosting of ECOC (Error correcting output coding) codes then after combine this code with SVM for the classification of age .when they applied Gabor feature, LBP, Feature fusion to ECOC_SVM then faced memory problem .For reducing these problem adopt PCA to reduce the dimension of LBP and Gabor features respectively before combing into single vector and achieved average accuracy is $85 \%$. Experiment on FERET and FG-NET Database.

Guodong Guo [4], presented that Is Gender recognition affected by human age? For solving this problem they used empirical studies on huge face database of more 8000 images with the ages. Based on these affects on human faces, LBP and Histogram oriented gradients (HOG) methods are used to evaluate for sex categorization with age variation. This paper also used bio-inspired feature for sex recognition.SVM classifier used for the classification of SEX. Accuracies for gender classification in age variations are $86.55 \%$ (Young), 95.03\% (Adult) and $89.04 \%$ (senior).

Ryotatsu Iga[5], developed an algorithm to estimate Gender and Age using (SVM) based on features like geometric arrangement and luminosity of facial images. The graph matching method with GWT method is used to detect the position of the face. GWT features, such as geometric arrangement color, hair and mustache are used for gender estimation. GWT features viz., texture spots, wrinkles, and flabs are used for age estimation.

Young H Kwon[6], presented classification from facial images. The primary features of the face are eyes, nose, mouth, chin, virtual top of the head and sides of the face are computed using ratios to identify young adults and seniors. In secondary feature analysis the wrinkle index computation is used to distinguish seniors from young adults and babies. A combination of primary features and secondary features determine face into one of the three classes viz., babies, young, adults and seniors.

\section{B. Gender classification: -}

In terms of gender classification, it is an easy task for humans but it is a challenging task in computer vision. Mostly, to classify the gender, face images are used, because people can easily cover their bodies with clothes.

Len Bui, Dat Tran[7],presented novel method for gender recognition by using $2 \mathrm{D}$ principal component analysis for extracting the feature vector and combine these 2D PCA with Support vector machine (SVM) discriminative method for classification. Experiments for this approach have been conducted on FERET data set. Average error rate $4.51 \%$. Huchuan Lu and Hui Lin[8], presented combination of the ellipse face images, Gabor filters, Adaboost learning and SVM classifier. Harr-like feature Gabor feature or ICA method are used to extract facial appearance information and achieved better performance.

Srinivas Gutta [9],presented improved classifier capability by using hybrid approach The hybrid approach consist of an ensemble of RBF networks and inductive decision trees. Experiment of this approach conduct FERET Database and achieved accuracy are $96 \%$ on the gender classification task and $94 \%$ on the ethnic classification task. C.F Lin [10], presented an approach based on fuzzy support vector machine with good generalization ability. The fuzzy membership function assigned to each input face feature data the degree of one human face is belonging to male or female face The aim of the fuzzification in FSVM is that different contributions to the learning of the decision surface.

B.Moghaddam[11],Used support vector machine(SVM) with radial basis function kernels to classify from low resolution 12*12 "Thumbnail" faces .They used 1755 faces from FERET face database to evaluate the classifier and achieved Classification accuracy of $96 \%$. R.Brunelli[12], used a set of 16 geometric features per image to train two competing networks with the radial basis function, one network for male and other for female and the classification rate was $79 \%$ on 168 training image show an error rate $21 \%$. Hui-chang lain[13],presented Multi-view gender classification considering both shape and texture information to represent facial images. The face area is divided into small regions, from which local binary pattern (LBP) histograms are extracted and concatenated into a single vector efficiently representing the facial image. Support Vector Machine (SVM) classifier is used for classification. Guillaume Heusch [14], proposed LBP as an image Pre-processing face authentication for illumination variations.LDA and HMM techniques are used for face authentication. Zehang Sun[15], Proposed gender classification from frontal facial images using genetic feature subset selection. Principal Component Analysis (PCA) is used to represent each image as a feature vector in a low-dimensional space. Genetic algorithms select a subset of features from the low-dimensional representation by disregarding certain eigenvectors that do not seem to encode important gender information. Bayes, Neural Network, SVM, and LDA classifiers are used and compared using Genetic Algorithm feature subset selection.

Ramesha K , K B Raja, Venugopal K R and L M Patnaik[16], proposed new feature based FEBFRGAC algorithm .The geometric features from a facial images are obtained based on the symmetry of the human faces and variation of gray levels, position of eye nose and mouth are located by applying the Canny edge operator. The gender is classified based on posteriori class probability and age is classified based on the shape and texture information using Artificial Neural Network. Gutta [17], Proposed an approach for recognizing the gender, ethnicity and age with facial images. Combination of Gabor filter, Adaboost learning and SVM classifier. Gabor filter banks and Adaboost learning are combined to extract key facial features of each pattern. Then used the Gabor+Adaboost features based SVM classifier to recognize the face image of each pattern.. Christian Ku "blbeck [18], proposed the new feature set local structure features computed from a $3 \times 3$ pixel neighborhood using a modified version of the census transform with four stage classifier .

Each classifier consists of a set of lookup-tables for feature weights. The training of a stage classifier is done using a version of the boosting algorithm. Yoav Freund [19], introduced 
the boosting algorithm AdaBoost, and explains the underlying theory of boosting and has also explained how to reduced generalization error, training error and relation to support vector machine

\section{Ethnicity Classification-}

Ethnicity is an important demographic attribute of human beings, and automatic face-based classification of ethnicity has promising applications in various fields and its identification present yet another challenge in face processing. Several authors have attempted this research problem as a challenge.

Hosoi, S. Takikawa, E. and Kawade, M [20] presented the Gabor wavelets transformation and retina sampling are combined to extract key facial features, and support vector machines that are used for ethnicity classification. Hui Lin, Huchuan Lu and Lihe Zhang[21], presented an MM-LBP (Multi-scale Multi-ratio LBP) method, which is a multimodal method for ethnicity classification. LBP (Local Binary Pattern) histograms are extracted from multi-scale, multi-ratio rectangular regions over both texture and range images, and Adaboost is utilized to construct a strong classifier from a large amount of weak classifiers. Yongsheng Ou, Xinyu $\mathrm{Wu}$, Huihuan Qian and Yangsheng Xu [22], presented the real time race classification system using PCA for feature generation and IDA for feature extraction. For classification they have been used new classifier (combination of SVM classifier).Experiment on FERET database with 750 face images and achieved $82.5 \%$.

In the light of the above literature review it can be concluded that classification of human attributes (age, gender and ethnicity) is not yet present with single classifier. This research is an attempt to build classification engine which will achieve the different goal like human gender, age and ethnicity.

\section{Proposed Methodology}

In the current work, we have presented an efficient system for human face detection and its attributes recognition like gender, age, ethnicity by single classifier using strong classifier which is collection of weak classifier.

The central contribution of this paper is the classification of attributes of the image search function that takes an arbitrary portion of the image in the space of states $\{-1,0,1\}$.

$$
H: u \rightarrow\{-1,0,1\} \quad(1)
$$

Where -1 - corresponds to one decision attribute label -1 (e.g., "women"), 1 - corresponds to another decision attribute label 1 (e.g. "man") and 0 - corresponds to abstaining from solutions (unknown).

Our approach is to maximize the use of "bionic" principles of construction of such systems, which have an analogy with the retinal receptive fields and visual cortex. In particular, we use simple tests derived from a nonlocal field of images that can be interpreted as "receptive fields", issuing a code description of the three segments the image and activate the "weak classifiers" and the ensemble of weak classifiers, combined in a committee or a strong classifier can be regarded as an analog artificial neurons located in the visual cortex. At the same time, developed and implemented system has a detection mode online recognition, and learning mode based on "past experience" of the system.

\section{A. Description of the prepared Embodiment.}

In the view of previously accomplished work in this direction, nobody has classified human attributes such as Gender, Age and Ethnicity with a single classifier. The overall architecture of the system is described below. We have divided our system in two subsystems. The first is the Facial attribute recognition system and the second is the Attribute classifier training system.

The Attribute recognition system includes a source of data (which may be a video camera or image viewer) as well as a face detection module for cropping face, an attribute recognition module for classifying attributes of the cropped face and a database for simultaneous storing processed images by the upper part of the architecture, i.e. Figure1 (a). In this mode Function (1) is used for processing the image fragment which is being reviewed, obtaining code description and decision-making. The Training subsystem Fig1 (b) includes a source database (which is united with the recognition system database) as well as tools for interaction with the operator, a teaching database and a training module. The overall objective of this subsystem is to build or upgrade Function (1) with further objective of achieving more accurate results in the future. The training system works with "previously acquired experience of the recognition system" which is stored in the image database and which forms a prototype of "short-term memory" in human brain while the "teacher" as an external power in relation to the system corrects the data so that the system is able to build an upgraded version of the classifier which is a prototype of "long-term memory" in human brain. An important feature of the described system is that it constantly updates its classifier. This fact allows the system to adapt to changing environmental conditions within the time and accumulate "acquired experience" in the structure of the strong classifier (1).

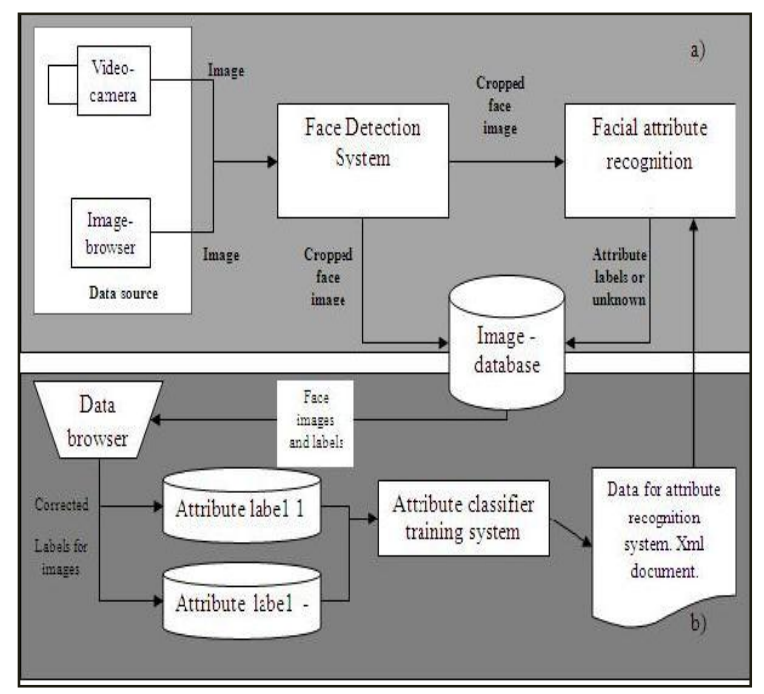

Figure1. Architecture of the recognition system attributes on the facial images a) and learning classifier system attributes b).

An original image which is obtained from a video camera or a viewer enters the face detection system. If a face is 
detected on the image, the detector cuts it out and stores it in a specialized image database. Then the facial image is sent to the attribute detector which assigns it a corresponding tag: attribute name or the label "unknown". Later on, using a special program (data browser), the user can check the tags which have been assigned by the attribute recognition system and, if necessary, correct them. Thus, a database of training and updating for the attribute classifier is formed. The attributes are formed using the training procedure and are used by the attribute classifier while the system is working in the recognition mode.

\section{B. Description of Attribute Recognition System}

Attributes recognition system is a modified strong classifier in the notation of Viola and Jones [21], consisting of a set of weak classifiers that work with data that are obtained from simple attributes derived from the original image fragment .In Figure 2 , we have shown the operation of recognition system, we set the size of the found face image in $30 \times 30$ pixels, and then convert the image to the integral form [20], to accelerate the computation of characteristic values obtained in this way the image is input to the strong classifier, which analyzes the image and takes one of three solutions.

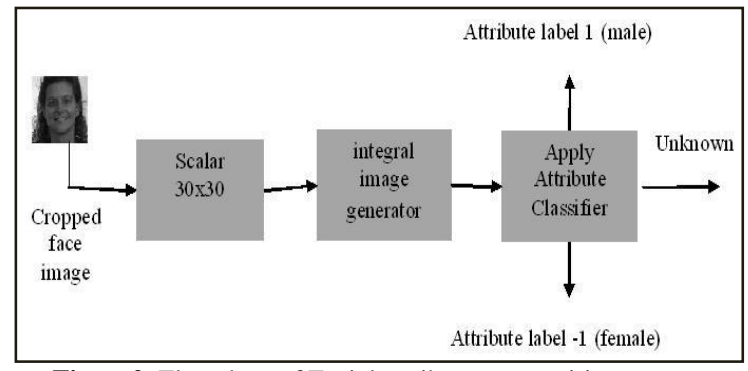

Figure2. Flowchart of Facial attributes recognition system

As shown in the figure, the strong classifier has three possible solutions: attribute label 1 , attribute label -1 and the unknown. Solution is defined in DSS (Decision Support System). Thus, it has a strong classifier committee structure, shown in the above figure.

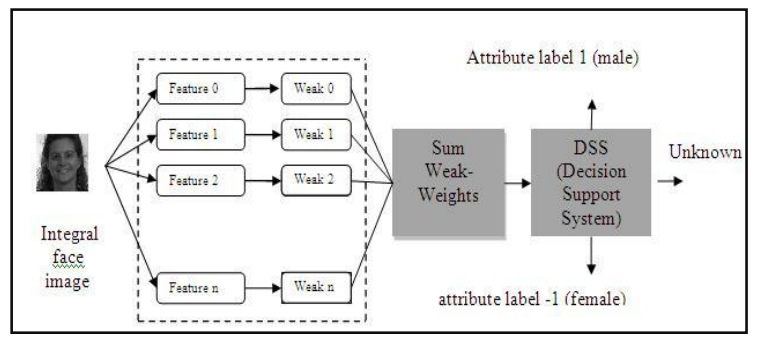

Figure3. The structure of the strong classifier

Simple tests, which are calculated from different parts of the image. Form the coded descriptions of these fragments, the code description to the inputs of weak classifiers, each of which shall decide exactly corresponding to the solution of a strong classifier or committee i.e. an attribute label, attribute label -1 and unknown. In addition, each weak classifier has its own weight, which is summed in the accumulator; the value goes to decision Support System, which makes the final decision on the recognition of the input image.
We consider all the elements of a strong classifier constructing, which includes forming code description of image fragments, the weak classifiers selecting and training.

\section{Non-local features based on the Modified Census Transform.}

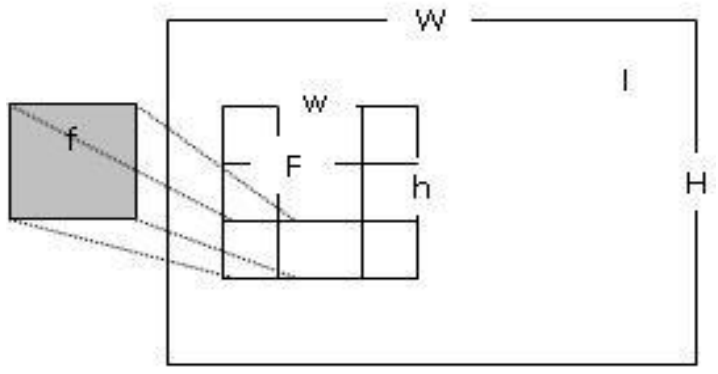

Figure4. Location attribute of the image.

Let there be $\mathrm{F}(\mathrm{w}, \mathrm{h})$ - a rectangular region image $\mathbf{I}$ of width $\mathrm{w}$ and height $\mathbf{h}$ pixels. We divide this Image land into 9 equal parts as shown in Figure4 and we analyze the average brightness of each area of the fragment $f \mathrm{u}$, comparing it with the average brightness of the fragment $\mathrm{F}$.

Determine the average brightness of the fragment $\mathrm{F}$ as follows:

$$
<I_{F}>=\frac{1}{w \cdot h} \sum_{x=x_{o}}^{x=x_{0}+w} \sum_{y=y}^{y=y_{0}+h} I(x, y)
$$

$I(x, y)$ - Brightness of the pixel with coordinates $\mathrm{x}, \mathrm{y}$.

Determine the average brightness $\left\langle I_{f^{n}}>\right.$ area $f^{n}$ area owned fragment $F$ followed as follows:

$$
<I_{f^{n}}>=\frac{9}{w \cdot h} \sum_{x=x_{i}}^{x=x_{i}+w / 3} \sum_{y=y_{j}}^{y=y_{j}+h / 3} I(x, y),
$$

For each area $f^{n}$ we encode the brightness of the following rule:

$$
c_{f^{n}}= \begin{cases}1, \text { if }<I_{f^{n}}>\geq<I_{F}> \\ 0, & \text { otherwise }\end{cases}
$$

Where $c_{f^{n}}$ - binary code of brightness for each area of the image fragment $\mathrm{F}$.

In accordance with (4) for each piece of the image we have a set of nine coded bits.

$c_{f^{0}} \ldots \ldots . . c_{f^{8}}$ Sequence which can be regarded as any $\mathrm{C}$ code image fragment ' $I$ '. Thus, any rectangular portion of the image, we can describe an integer $\mathrm{C}$, having a 9-bit word length in the decimal system $0 \leq C<512$. 


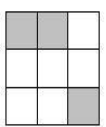

$\mathrm{C}=385$

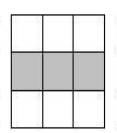

$\mathrm{C}=56$

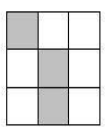

$\mathrm{C}=274$

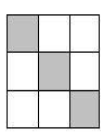

$\mathrm{C}=273$

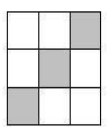

$\mathrm{C}=84$

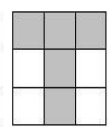

$\mathrm{C}=466$

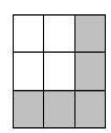

$\mathrm{C}=79$
Figure5. Pattern of the codes $\mathrm{C}$, Where the white area of the image coded with 1 and gray coded with 0 .

\section{Training a weak classifier}

We shall consider a weak classifier as a function that takes the code $\mathrm{C}$, the resulting non-local Modified Census Transform in the solution space:

$$
h: C \rightarrow\{-1,0,1\},(5)
$$

Since the feature value MCT has an integral nature, the function of the decision for the weak classifier (5) is simple lookup table, which contains 512 items that match the codes C.

E. To obtain such a lookup table, we have used the following procedure to study the weak classifier:

1) Suppose you have a database of images each of which is marked attribute label $(-1,1)$.

2) Let there be two histograms h1 [512] and h2 [512]. Each contains the distribution of the codes featured image database. Where hl corresponds to the distribution of the codes for a subset of data from the attribute label $=1$, and $h 2$ corresponds to the distribution of codes for a subset of data from the attribute label $=-1$.

3) Then the formation of the decision rule is as follows (6):

$$
h[C]=\left\{\begin{array}{l}
1, h 1[C]>h 2[C] \\
-1, h 1[C]<h 2[C],(6) \\
0, h 1[C]=h 2[C]
\end{array}\right.
$$

Thus, obtained is a weak classifier is fully compliant with our system and its properties are determined exclusively by the input data set.

\section{$F$. The learning procedure is a strong classifier}

We seek a strong classifier (1) as follows:

$$
H(x)=F\left[\sum_{i=1}^{n} \alpha_{i} h_{i}(x)+T\right]
$$

Where $\mathrm{x}$ - considered fragment, $\mathrm{F}[\mathrm{v}]$ - a nonlinear function of making solutions, $h_{i}$ - weak classifier (6), $\alpha_{i}$ - weight of a weak classifier, $\mathrm{T}$ - the threshold of decision-making and $\mathrm{n}$ the number of weak classifiers in the committee. Here we use the following form of the nonlinear function $\mathrm{F}$ [v], which is implemented in the DSS (Decision Support System)

$$
F[v]=\left\{\begin{array}{c}
1, v>0 \\
-1, v<0 \\
0, v=0
\end{array}\right.
$$

$\Gamma$ de 1 corresponds to the address "attribute label 1", -1, consistent with the decision "attribute label -1 " and 0 corresponds to the solution of "unknown".

As we all aware with the well-known amplification method is one way to implement a class of associative machines. Classifiers is operating on the basis of the gain, study the examples belonging to very different distributions. To build a strong classifier (7), we are using the quite famous procedure AdaBoost, is widely known in the literature.

The purpose of using this algorithm is to find the final mapping function or hypothesis $\mathrm{H}$, which will have low or acceptable level of error in this subset of labeled examples of learning. From other algorithms enhance AdaBoost differs as follows:

- AdaBoost adaptively adjusts the error of weak hypotheses returned by the weak learning model. In our case, the decision-making functions (6). Due to this property the algorithm got its name.

- Limiting performance AdaBoost depends on the performance of weak learning model (6) and only those distributions that are actually formed in the learning process.

A typical graph of the learning process of a strong classifier using AdaBoost procedure is shown in Figure 6. The initial data used in a database of men (attribute label 1) and women (attribute label -1), containing 1200 images.

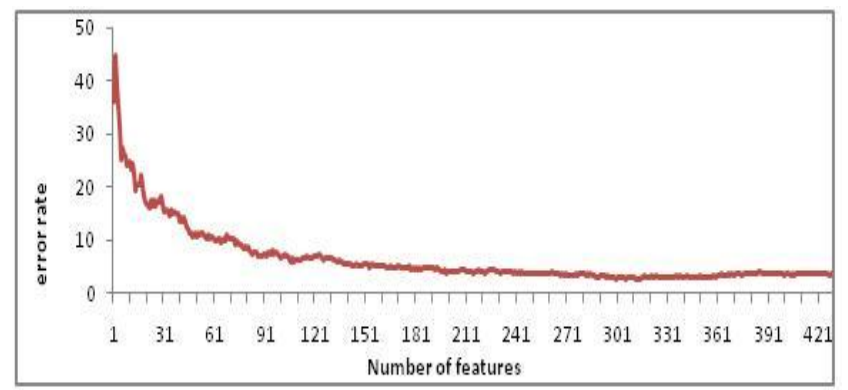

Figure6. Dependence of the overall recognition error of a strong classifier the number of features selected by AdaBoost procedure in the learning process based on data from men-women, containing 1200 images.

\section{EXPERIMENTAL PROCEDURE}

In order to estimate the performance of the proposed system, we collected a set of images of human faces from the World Wide Web, FERET and FG-NET.

To study the recognition of attributes, built on a strong classifier (7), we chose several databases.

FERET database to conduct research on the recognition of men and women, database FG-NET for experiments on the recognition of age and a database $1 \mathrm{fw}$ _funneled to conduct experiments on the recognition of race. Database Features in the following table:

Table1. Databases Feature.

\begin{tabular}{lllll}
\hline Database & $\begin{array}{l}\text { Attribute } \\
\text { label 1 }\end{array}$ & $\begin{array}{l}\text { No. of } \\
\text { Images }\end{array}$ & $\begin{array}{l}\text { Attribute } \\
\text { label -1 }\end{array}$ & $\begin{array}{l}\text { No. of } \\
\text { Images }\end{array}$ \\
\hline FERET & Male & 785 & Female & 461 \\
FG-NET & Adult & 225 & Child & 390 \\
RUS-IND & Europeans & 910 & Asians & 646 \\
& & & & \\
\hline
\end{tabular}


For each image, face detection system finding the face and its location is stored in an internal database. Figure 1. No manual adjustments of the detector shall not be satisfied, except for removing artifacts search, i.e. sites background, taken for a person. In addition to these databases, for the age recognition experiment we have used our own database of photos which has been collected from World Wide Web contains 800 images of adult and 600 face images of children.

\section{A. The choice of size and scale the face image analysis}

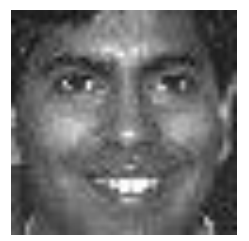

Figure7. The characteristic image of the face, resulting detector

The detector has used in this investigation cuts the faces for analyzing from initial picture without hair style and other face features, which may be important for face classification. There is very interesting to investigate by use of computer simulations how the results of face classification depend on these circumstances. For this aim we realize experiments series for changing sizes of images rectangle and the size of the human face in the rectangle considered.

We experimented with nine different sizes of images 24, $27,30,33,36,39,42,45$ and 48 pixels. By increasing the size of pixels we have not any significant in the accuracy of the classifier At the same time, learning increases significantly with increasing size of the images, since greatly increased the number of features and weaknesses of the classifiers involved in the training process. On this basis, we recorded the size of the training images by thirty pixels. This changes the scale of the face, increasing the size of the rectangle cut by $10 \%, 20 \%$ and $30 \%$, respectively, we get the following scale: $100 \%$ - the initial size of human obtained by the detector are $90 \%, 80 \%$ and $70 \%$. Further size reduction leads to significant areas of the background of the image.

Figure 8. Illustrates the changes in the scale of a person in the picture, depending on the cut size of the rectangle.
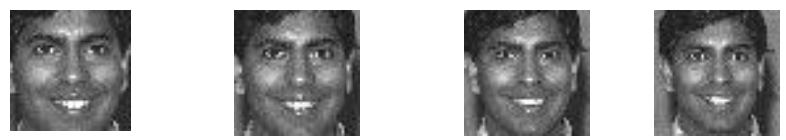

$100 \%$ $90 \%$

$80 \%$

$70 \%$

Figure8. Zoom in person, depending on the degree of increase found detector rectangle.

To test the dependency of the sharpness of the classification on the scale of the person we used a database FERET (Table 1). Testing results are shown in Figure

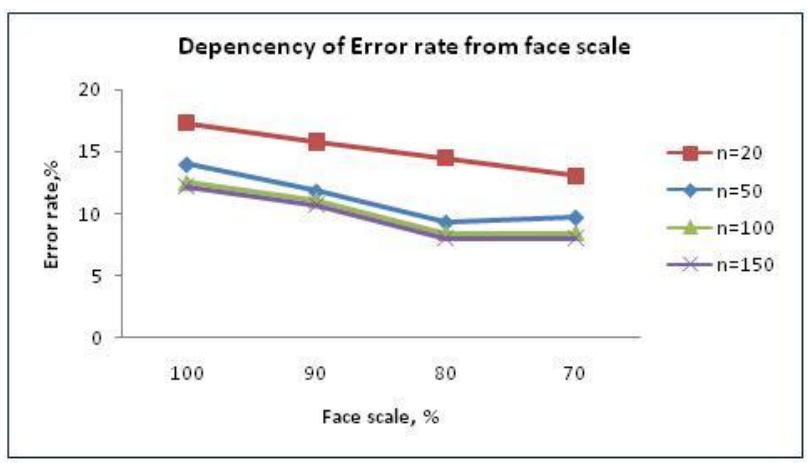

Figure9. Dependency of recognition errors on the scale person in the picture.

The above graph shows that, the face image with the different scale improves the accuracy of the system. The results are shown for different numbers of selected features. Thus in all further experiments, we used an image size of 30x30 pixels, and set the scale of human equal to $80 \%$ on the scale found by the detector.

\section{B. Education and research Classifications.}

All classifiers were trained and constructed as described above. For their building to used databases that are described in Table 1. Performed several dozen training cycles classifiers on random samples taken from these databases. $70 \%$ of the data used for training, the remaining 30\% of the data used for testing. We investigated the following properties of classifiers:

$>$ Dependency of recognition errors on the number of the selected algorithm AdaBoost weak classifiers

$>$ Dependency of the ROC for different values of T (7). With regard to the test subset of the original data. ROC curves were constructed for each class individually recognizable.

In addition to these values fixed minimum error values for each of the data sets as learning and testing. A full description of the experiments can be found in the section Results.

\section{EXPERIMENTAL RESULTS}

\section{A. Classification Performance of the attributes classifier}

\section{1) GENDER Classifier.}

For training and testing database were used FERET, comprising 785 men and 461 images of a woman. 58 cycles were carried out for constructing a classifier on different subsets selected from the initial data at random. As the proportion of $70 \%$ of training subset, $30 \%$ of the testing subset. The average value of recognition errors, as in the test subset, and in the tutorial can be seen in

We carried out 300 cycles of updating the classifier, for each of the random data sets. It was found that the value of recognition errors on a test set of changes little after 250 weak 
classifiers, while on the training dataset, the value of recognition errors continued to fall.

Averaged dependence of the FRR (FAR) for different values of $\mathrm{T}$ (7), shown in Figure 11.
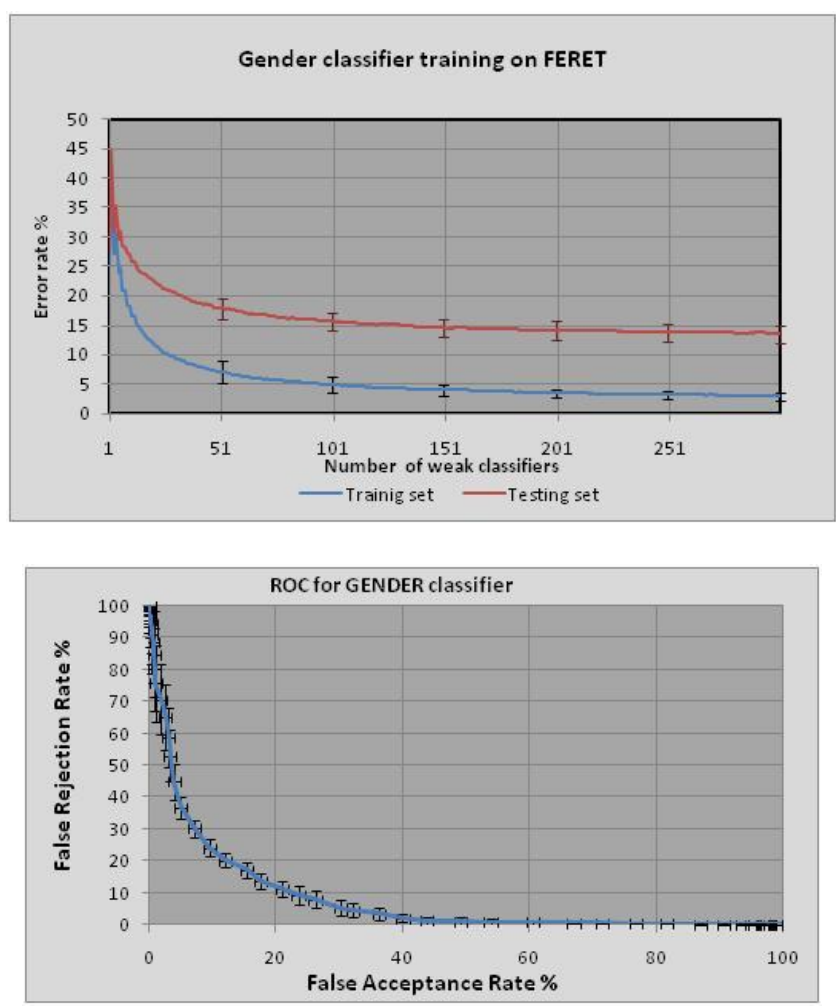

Figure11. Average ROC for GEDNDER classifier, based on FERET.

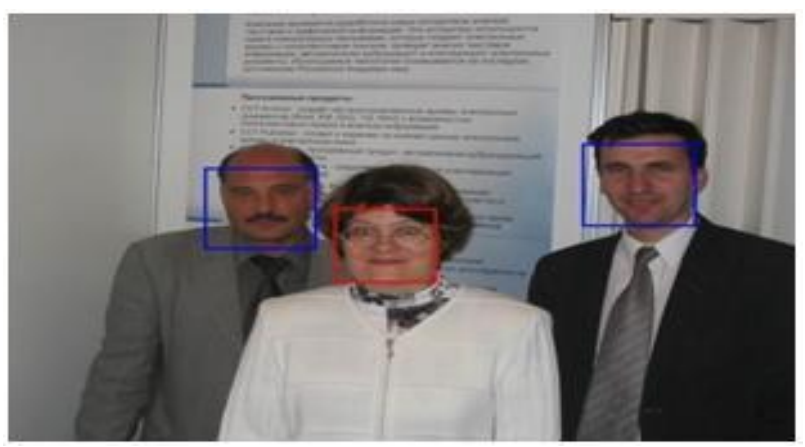

Figure12. Result of Gender Classifier Blue- MALE, Red-FEMALE

\section{2) AGE Classifier.}

For training and testing database ware used FG-NET and the database collected during the study. The database includes 905 images of children and 722 adults image. The database is significantly more complex compared to a database FERET because the quality of images used in the studies of a lower light conditions and angle are not controlled. As in the previous case was carried out 58 cycles of building a classifier on different subsets selected from the initial data at random.

The proportion of $70 \%$ of training 1 subset, $30 \%$ of the test subset. The average value of recognition errors, as in the test subset, and in the tutorial can be seen in Figure 13.

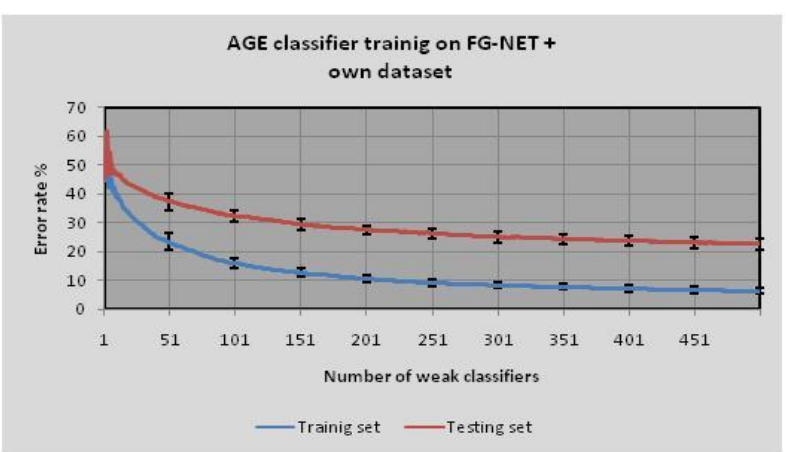

Figure13. Training process for AGE classifier.

We carried out 500 cycles of updating the classifier for each of the random data sets. It was found that the results of the classifier is somewhat worse than on the database FERET, but no trend for an end to learning have been identified. Averaged dependence of the FRR (FAR) for different values of T (7), shown in Figure 14.

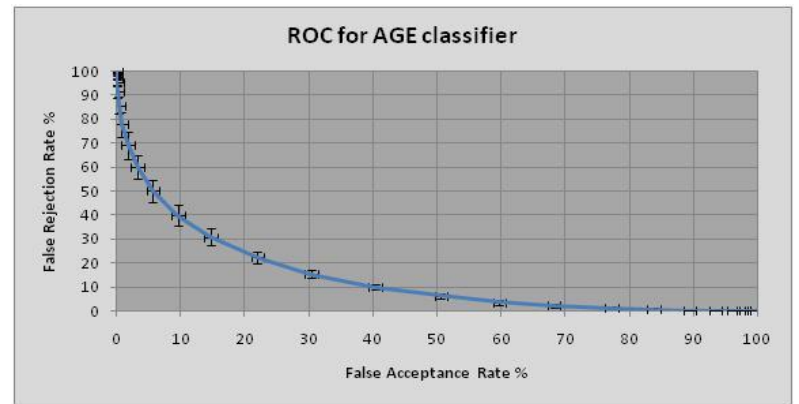

Figure14. Average ROC for AGE classifier, based on FG-NET

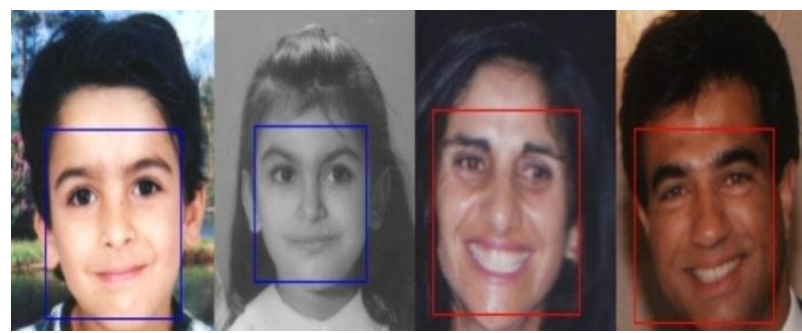

Figure15. Classification of age attributes on FG-NET. Blue-CHILD, Red -ADULT

\section{3) Ethnicity Classifier}

To train our classifier for recognition of ethnicity we have used Indian Face Database [22] and the database collected during the study in Applied Physics Institute RAS. The database includes 910 images of Europeans and 722 Indians. As in the previous case was carried out 20 cycles of building a classifier on different subsets selected from the initial data at random. The ratio of $70 \%$ of training subset, $30 \%$ of the test subset. The average value of recognition errors, as in the test subset, and in the tutorial can be seen in Figure 16.

We carried out 200 cycles of updating the classifier for each of the random data sets. It was found that the results of the classifier are somewhat better than on the database FERET. Averaged dependence of the FRR (FAR) for different values of $\mathrm{T}$ (7), shown in Figure 17. 


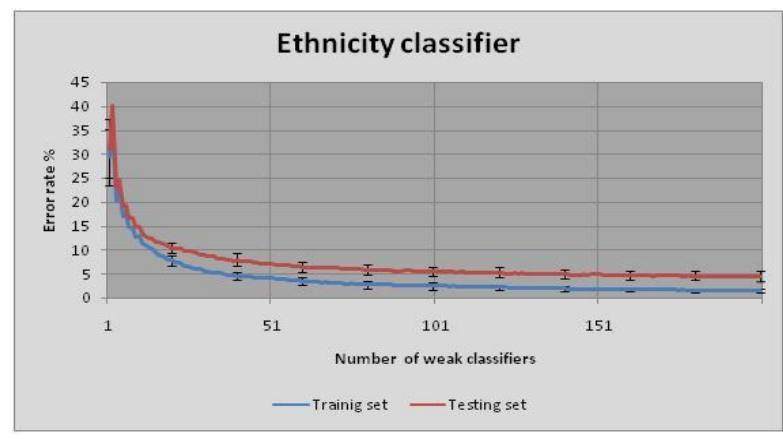

Figure 16.Training process for Ethnicity classifier.

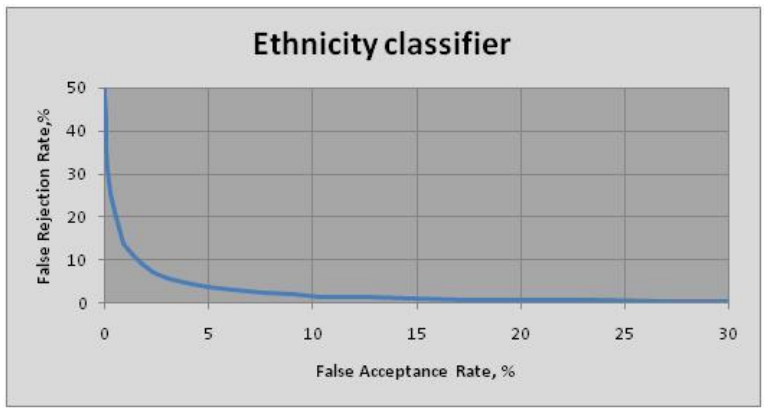

Figure 17. ROC for Ethnicity classifier.

As we all know that, a system with artificial intelligence cannot do work like human natural intelligence but still researches are moving towards the automation or minimize the human efforts. Therefore face recognition and computer vision are the active area of the research.

TABLE 2- RECOGNITION ACCURACY ACHIEVED

\begin{tabular}{cc}
\hline Human Attributes & Achieved Accuracy \\
\cline { 2 - 2 } Gender(Male, Female) & $89 \%$ \\
Age(Adult, Child) & $80 \%$ \\
Ethnicity & $96 \%$ \\
\hline
\end{tabular}

\section{FINDINGS \& DiSCUSSION}

In the present study was formulated and investigated neuron algorithm to automatically construct a classifier that separates the two-dimensional visual image into two classes according to some binary characters. Algorithm is formed from the available data and is quite universal because it uses a number of general principles of teaching:

a) Partitioning an existing database on two samples - a training and test,

b) Creation of a "strong" classifier in the form of a committee of the primary "weak" classifiers, whose voices in the decision on the committee are recorded in accordance with rating scales for each,

c) Analysis of errors of the classifier on the number of members on the committee,

d) Control of the invariance result with respect to partition the database into training and test samples.

Established authors program allows an effective and unique tool for computational and experimental determination of optimal values of those parameters, to find that speculative way is not possible. The result is determined by the brute force of several options, the calculation of total errors for each of them, and selection of options with the least error. As examples, which are important for practical applications in this paper, the study addressed the question of optimization of two parameters of the problem:

\section{a) The scale of images used to create a classifier \\ b) The size of the face image on the compared fragment.}

The results are shown in Fig. 9.

As shown in Fig. 10.12, after the number of items the committee is more than 50 error and the learning and test sample subside slowly, and the error on the test sample is 3-5 times less than on learning. Such behaviors of the curves are not exactly conventional ideas and are a new result that requires further study. It is not clear as to why the error curve for training database does not tend to zero.

\section{CONCLUSION \& FUTURE WORK}

The study showed versatility and promise of the proposed approach to the description of such complex objects as the human face, but at the same time allowed to see the boundaries of the new method. First, a simple increase in the number of elements in does not automatically lead to decay to zero errors, not only for the test sample, but also the learning part of the database. This is indicative of the fact that the quality of the classifier, constructed by the proposed method can and should be improved.

The above findings allow us to identify ways of further improving the method of Classification and enhance its effectiveness.

1. Firstly, we should construct a two-level classifier, which will be a committee of "strong" classifiers trained on different data. Such a qualifier in the second level can be built from firstlevel classifier for the scheme, similar to how the first-level classifiers ("strong" classifiers) are constructed from the original zero-level classifiers (weak classifiers).

2. Secondly, to improve the classification results should lead to introduce an additional threshold and extending the range of values of the internal variable, where the classifier says "I do not know.

Application of the elements with two thresholds which have three positions in voting (yes, no, I do not know), allows to build consistent and achieve better results in classification, if we can effectively address their learning according to a reasonable time.

Third, a wide enough field to improve the level of classification should be receptive to the use of elements of another type, than used here type"census". It is possible use elements with Gabor functions receptive structures or Haar functions receptive structure. Created soft tools allow make computer experiments for these variants comparing and choosing the best.

\section{ACKNOWLEDGEMENT}

The authors would like to thank Department of Science and Technology, Government of India and Russian Foundation of 
Basic research for supporting the research under Indo-Russian bilateral arrangement through the project sanction number DST/RFBR/P-69

\section{REFERENCES}

[1] Yi-Wen Chen ; Meng-Ju Han ; Kai-Tai Song ; Yu-Lun Ho.: Image-based age-group classification design using facial features. In: System Science and Engineering (ICSSE), 2010 International Conference on 2010, Page(s): 548 - 552.

[2] Asuman GÜNAY, Nabiyev, V.V.: Automatic Age Classification with LBP. In: Computer and Information Sciences, ISCIS '08. 23rd International Symposium 2008 , Page(s): 1 - 4

[3] Jian-Gang Wang; Wei-Yun Yau; Hee Lin Wang.: Age Categorization via ECOC with Fused Gabor and LBP Features. In: Applications of Computer Vision (WACV), 2009 Workshop 2009, Page(s): 1 - 6

[4] C.F. Lin, S.D Wang .:Fuzzy Support vector Machines. In: IEEE Transactions on Neural network, Vol.13,No.2,pp.464-471,Mar.2002

[5] Young H. Kwon and Niels Da Vitoria Lobo.: Age Classification from Facial Images. In: Journal of Computer Vision and Image Understanding, vol. 74, no. 1, pp. 1-21, April 1999

[6] Fukai, H.; Takimoto, H.; Mitsukura, Y.; Fukumi.: Age and gender estimation by using facial image M. In: Advanced Motion Control, 2010 11th IEEE International Workshop 2010 , Page(s): 179 - 184

[7] Guillaume Heusch,Yann Rodriguez and Sebasttien Marcel.: Local Binary Patterns as an Image processing for face Authentication. In: Proceedings of seventh International conference on Autometic Face and Gesture Recognition,pp.6-14.April 2006.

[8] Len Bui; Dat Tran; Xu Huang; Chetty, G.: Face Gender Recognition Based on 2D Principal Component Analysis and Support Vector Machine. In: Network and System Security (NSS), 2010 4th International Conference 2010 , Page(s): 579 - 582

[9] Huchuan Lu; Hui Lin.:Gender Recognition using Adaboosted Feature. In : Natural Computation, 2007. ICNC 2007. Third International Conference on Volume: 2 2007, Page(s): 646 - 650

[10] B.Moghaddam and M.H Yang.:Learning Gender with support faces. In: IEEE Transactions on Pattern Analysis and Machine Intelligence, Vol.24, No.5,pp. 707-711,2002.

[11] R.Brunelli and T.Poggio.:HyperBF Networks for gender classification.In: Proceedings of the DARPA Image Understanding Workshop,Pages 311314.1992.

[12] Hui-Cheng Lian and Bao-Liang L.: Multi-view Gender Classification Using Local Binary Patterns and Support Vector Machines. In: Advances in Neural Networks - ISNN 2006.

[13] Hui Lin; Huchuan Lu; Lihe Zhang.: A New Automatic Recognition System of Gender, Age and Ethnicity.In: Intelligent Control and Automation, 2006. WCICA 2006. The Sixth World Congress on Volume: 2 ,2006 , Page(s): 9988 - 9991

[14] Christian Ku blbeck *, Andreas Ernst.: Face detection and tracking in video sequences using the modified census transformation. In: Image and Vision Computing 24 (2006) 564-572

[15] Ramesha K et al.: Feature Extraction based Face Recognition, Gender and Age Classification. In: (IJCSE) International Journal on Computer Science and Engineering, Vol. 02, No.01S, 2010, 14-23

[16] Hosoi, S.; Takikawa, E.; Kawade, M.: Ethnicity estimation with facial images Automatic Face and Gesture Recognition. In: Proceedings. Sixth IEEE international Conference 2004, Page(s): 195 - 200
[17] Gutta, S.; Wechsler, H.:Gender and ethnic classification of human faces using hybrid classifiers Neural Networks. In: International Joint Conference on Volume: 6,1999 , Page(s): 4084 - 4089 vol.6

[18] Zehang Sun, George Bebis, Xiaojing Yuan, and Sushil J. Louis.: Genetic Feature Subset Selection for Gender Classification: A Comparison Study.In: IEEE Workshop on Applications of Computer Vision, pp.165170, 2002.

[19] Guodong Guo; Dyer, C.R.; Yun Fu; Huang, T.S.: Is gender recognition affected by age? In: Computer Vision Workshops (ICCV Workshops), 2009 IEEE 12th International CONFERENCE 2009, Page(s): 2032 2039

[20] Y. Freund and R. E. Shapire.:A short introduction to boosting. In: Journal of Japanese Society for Artificial Intelligence, pages 771-780, 1999

[21] Paul Viola, Michael Jones.: Rapid object detection using a boosted cascade of simple features. In: Proceedings of the IEEE Conference on Computer Vision and Pattern Recognition, 2001.

[22] Indian Face hatabase.//viswww.cs.umass.edu/ vidit/IndianFaceDatabase/

\section{AUTHORS PROFILE}

Nikolai Sergeevich Bellustin received his graduate degree from radio physics faculty the University of Nizhny Novgorod. He defended the candidate thesis on the different theme. He defended his doctoral thesis. At present time he is a professor of Nizhny Novgorod technical university. The author has worked on various areas of scientific interests:

Yuri Dmitrievich Kalafati graduated from the Moscow physico-technical institute $\mathrm{He}$ defended the candidate thesis on the different theme. He defended his doctoral thesis. The author has worked on various areas of scientific interests:

Olga Vladimirovna Shemagina received his graduate degree from radio physics Nizhny Novgorod state university in 1996. He is the author of 15 printed works. Area of scientific interests: image processing and Computer vision.

G. Yakhno graduated from radio physics faculty Nizhny Novgorod state university in 1969. He defended the candidate thesis in 1977, on the theme "the Structure of collective activity in the excitable media". He defended his doctoral thesis in 1999 on the theme of "Transformation of the information in neuron systems". At present time he is a professor of Nizhny Novgorod state university. The author has work more than 150 areas of scientific interests: nonlinear dynamics in cognitive research.

Alexander Telnykh received his graduate degree from radio physics Nizhny Novgorod state university. He defended the candidate thesis in 2009 on the theme "Mathematical models neuron-like environments for the development of systems for the detection and recognition of objects set of classes". The author has work more than 30 areas of scientific interests: image processing, computer vision etc

Andrey V. Kovalchuck graduated from radio physics faculty the University of Nizhny Novgorod in 2006 is a graduate student. The author has worked on more than 18 areas of scientific interests: image processing, computer vision.

Abhishek Vaish received his $\mathrm{PhD}$ from Indian Institute of Information Technology, Allahabad India. He has done various researches on security domain.

Pinki Sharma received her M.Tech (CSE) degree from Bansathali University, India and she is currently Research Scholar at Indian Institute of Information Technology, Allahabad. 